that there is no such thing as a focal respiratory centre. The respiratory neural mechanism is an integrative one, the integration taking place at several levels. The reticular formation in the brain system is one of the primary regions for this integration.

G. W. T. H. Fleming.

Convulsive Manifestations in Huntington's Chorea. (Fourn. of Nerv. and Ment. Dis., August, 193I.) Notkin, $\mathcal{F}$.

The author, after reviewing the literature, presents a single case of his own, and points out that the pathological changes are not confined to the caudate nucleus, but are found in the cortex, in the prefrontal gyrus, frontal lobe, and sometimes in the parietal, temporal and occipital lobes. In the literature, in ten instances the convulsive attacks preceded the chorea, in six they appeared during its course, and in four they became manifest about the same time as the chorea. The author concludes that there is some relationship between Huntington's chorea and the idiopathic group of convulsive states.

G. W. T. H. Fleming.

\title{
6. Pathology.
}

Injury and Repair within the Sympathetic Nervous System. I. The Preganglionic Neurons. (Arch. of Neur. and Psychiat., September, I93I.) Tower, S. S., and Richter, C. P.

The authors operated on twenty-six cats, and cut the preganglionic sympathetic nerve-fibres on the right side prior to their entry into the stellate ganglion. After the operation there was immediate elimination of the spontaneous waves in the skin potential and of the galvanic skin response, combined with an immediate increase in skin resistance. The resistance of the skin reached a peak many times the maximum normal figure, and then fell slowly and with fluctuations; between the third and seventh weeks, as skin resistance again approached a normal figure, the action currents of the skin reappeared. The authors are of the opinion that the galvanic skin response is a far more delicate test for the presence of sympathetic innervation than the gross observation of the activity of the sweat-glands.

G. W. T. H. Fleming.

The Blood-Cerebro-spinal Fluid Barrier in Manic-depressive Psychosis. (Arch. of Neur. and Psychiat., October, 1931.) Rothschild, W., and Malamud, $W$.

The authors use Hauptmann's modification of 'the original Walter's method for estimating the ratio of distribution of fbromide, and investigated 100 cases of manic-depressive psychosis and 28 of involutional melancholia. Amongst the former group $35 \%$ 
showed an increased passage of bromide into the spinal fluid, $53 \%$ showed a normal ratio, and $41.5 \%$ of the depressed cases and $28.6 \%$ of the maniacal cases showed low ratios. Cases of agitated depression all showed low ratios. As the psychosis subsided the ratios moved towards the normal value. In involutional melancholia, $35 \%$ showed low ratios, and $56.5 \%$ a normal ratio.

The authors also investigated the distribution of calcium, sugar and chloride in a number of these patients. They found the distribution of calcium abnormal in $33.3 \%$ of the cases, almost all of which showed normal values for bromide. There was a tendency for the cases with low bromide ratios to show, as a group, a slightly greater passage of sugar into the spinal fluid than the group with normal or high bromide ratios. The chloride ratio of distribution was normal in manic-depressive insanity.

G. W. T. H. Fleming.

The Ganglioneuromas of the Central Nervous System. (Arch. of Neur. and Psychiat., September, 1931.) Alpers, B. F., and Grant, F. C.

The authors discuss the twenty-one cases reported in the literature, together with one of their own. Twelve of these cases occurred at the base of the brain, and nine in the cerebral hemispheres. Of those in the cerebrum the majority were in relation to the temporal lobe. The ganglioneuroma is much more common in young persons, the age in this series varying from eleven to forty years. Amongst the clinical symptoms, epilepsy is very common. In practically every case there was some connection with the brain substance, showing that fundamentally the tumours are infiltrating. Many of the tumours have large cysts together with other smaller cysts, probably of a degenerative nature. The tumours are composed of ganglion cells in varying numbers and different stages of development, of neuroglia cells and fibres and, in some instances, of nerve-fibres, which are usually unmyelinated. In some tumours the ganglion cells are almost entirely immature, and in most of them the ganglion cells fall short of complete maturity. The usual site is the region of the diencephalon.

G. W. T. H. Fleming.

\section{Mental Deficiency.}

Sex-linked Inheritance in Mental Deficiency. (Amer. Fourn. Psychiat., September, 1931.) Rosanoff, $A$. F.

The records of 95 pairs of twins were studied. One or both in each pair had mental deficiency. There were 35 monozygotic and 60 dizygotic pairs. Mental deficiency seems to be consistently more common in males than in females, and this was most strikingly shown in opposite-sex twins. Girls make a slightly better showing 